Einführung in das Schwerpunktthema

\title{
Transdisziplinäre ökologische Wirtschaftsforschung
}

$\mathrm{T}$ Von Uwe Schneidewind ransdisziplinarität wird immer mehr zu einer Kernforderung in der Nachhaltigkeitsdebatte: Dies gilt für die wissenschaftliche Diskussion in gleicher Weise wie für die Forschungsförderung, die Konzipierung neuer Umweltstudiengänge sowie die Anforderungen von Kunden in Beratungsprojekten bei Unternehmen und in der Politik.

Was verbirgt sich hinter der Idee der Transdisziplinarität? In der wissenschaftstheoretischen Diskussion ist damit Forschung gemeint, die sich an konkreten lebensweltlichen Problemen orientiert und für diese Probleme Antworten sucht. Nicht das Überwinden von Disziplingrenzen an sich (Interdisziplinarität) steht im Vordergrund, sondern der Problembezug. Die Interdisziplinarität jeder transdisziplinären Forschung ist vielmehr ein Beieffekt. Dabei ist die Abgrenzung zwischen Inter- und Transdisziplinarität in der wissenschaftstheoretischen Diskussion nicht unumstritten. Einige Autoren benutzen beide Begriffe auch synonym (1).

Für den Schwerpunkt des vorliegenden Heftes stand der Leitbildcharakter des Problembezugs im Vordergrund und hat zur bewußten Wahl des Begriffs Transdisziplinarität gefiuhrt. Denn die ökologische Wirtschaftsforschung ist ein für ein in diesem Sinne verstandenes transdisziplinäres „Wissen-Schaffen“ prädestiniertes Feld. Hier gilt es Antworten auf Fragen zu finden, die jeden von uns, die Unternehmen, die die Gesellschaft als Ganzes brennend drücken. Viele derjenigen, die im Kontext der VöW oder in ökologischen Forschungsinstituten „Wissen schaffen", sind über diesen konkreten Problembezug zu ihrem Themenfeld gestoßen. In der Wissens-Praxis fangen disziplinäre Leitplanken aber in der Regel den Forscher schnell wieder ein - trotz aller transdisziplinären Ideale. Die Fragen, wie sich Transdisziplinarität mit Leben fiillen läßt und wo die konkreten Chancen und Grenzen eines solchen Konzeptes liegen, waren daher Leitfragen der vöW-Jahrestagung 1997 in Berlin (2).

Der Schwerpunkt dieser Ausgabe von „Ökologisches Wirtschaften“ greift die Diskussionen im
Kontext der Jahrestagung wieder auf. Er zeigt, mit welchen Konzepten versucht wird, dem Transdisziplinaritätsanspruch in der Nachhaltigkeitsforschung gerecht zu werden (Marc Mogalle, Jürg Minsch), er illustriert in prägnanten Thesen, vor welcher Herausforderung gerade die ökologisch orientierten Wirtschaftswissenschaften bei der Überwindung ihrer disziplinären Begrenzung stehen (Adelheid Biesecker), er macht deutlich, wie ein konsequenter Empiriebezug das methodische Fundament für gute transdisziplinäre Forschung darstellt (Martin Jänicke) und zeigt, daß eine Brückenfunktion zwischen den Disziplinen aber auch von konkreten theoretischen Konstrukten wie dem der „Institution“ erfüllt wird (Uwe Schneidewind u.a.). Das Heft zeigt weiterhin an Beispielen, wie transdisziplinäre Ansätze in der Lehre (Christoph Schwarzer, Uwe Schneidewind) und in der Unternehmenspraxis ausehen können (Arnt Meyer, Frank Riemenschneider).

\section{vöW und Transdisziplinarität}

Den Themenschwerpunkt dieses Heftes versteht die VÖW nach der Jahrestagung als einen weiteren konkreten Meilenstein, um die Diskussion und das Praktizieren von Transdisziplinarität im Verein voranzutreiben.

Was heißt die Forderung nach Transdisziplinarität für die VÖW? Transdisziplinarität ist nicht nur eine konkrete Herausforderung an die inhaltliche Arbeit von Forschern, Unternehmenspraktikern und Beratern. Sie benötigt auch neue Netzwerke, in denen dieser Forschungszugang praktiziert wird. Die bestehenden Wissenschaftsverbände und die von Ihnen publizierten Zeitschriften sind in der Regel disziplinär organisiert. Sie bieten nur eine ungenügende Plattform für transdisziplinäres Schaffen von Wissen. Die VöW ist nun in zweifacher Hinsicht prädestiniert, zu einer institutionellen Plattform zu werden, die diese Lücke fuillt:

- Sie versammelt WissenschaftlerInnen aus Universitäten und anderen Forschungsorganisationen, PraktikerInnen aus gesellschaftlichen Kontexten und Studierende unter ihrem Dach.
Diese Zusammensetzung gewährleistet den problembezogenen und lebensweltlichen Bezug ihrer Arbeit. Den Briickenschlag zwischen in der Wissenschaft und in der Praxis Tätigen in der zukünftigen Arbeit zu stärken, ist daher eine wichtige Herausforderung für den Verein. Gerade dem Absolventenkreis kommt in diesem Kontext eine hohe Bedeutung zu.

- In der vöw sind neben Betriebs- und VolkswirtInnen immer schon auch Mitglieder aus anderen (Gesellschafts-)Wissenschaften organisiert gewesen. Mit dem (Wieder-)Einzug von Martin Jänicke in den VöW-Vorstand hat dies auch auf Vorstandsebene seinen Niederschlag gefunden. Dieses Potential, ForscherInnen unterschiedlicher Disziplinen, die eine gemeinsame inhaltliche Herausforderung - eben ökologisches Wirtschaften - verbindet, zu organisieren, gilt es in Zukunft ebenfalls auszubauen. Die Ausgestaltung der zukünftigen Jahrestagungen, der Einbezug von Partnerorganisationen wie der Initiative Psychologie und Umwelt z.B. in das Programm der Studierendenkreisakademien, aber auch eigens konzipierte interdisziplinäre Veranstaltungen (wie der letztjährige IPUvöW-Workshop zur Mitarbeiterbeteiligung im betrieblichen Umweltschutz) sind wichtige Schritte in diese Richtung.

Wir hoffen, mit diesem zum ersten Mal seit langer Zeit wieder federführend von der vöw gestalteten Schwerpunkt im Informationsdienst Ökologisches Wirtschaften - der ja gemeinsames Organ von IÖW und VöW ist - viele Diskussionsanstöße zu geben.

\section{Anmerkungen}

(1) Die Unterscheidung der beiden Begriffe wurde u.a. von Mittelstraß eingeführt. Vgl. z.B. Mittelstraß, J.: Interdisziplinarität oder Transdisziplinarität?, in: Hieber, L. (Hrsg.): Utopie Wissenschaft. München 1993. Für die Feinheiten und Hintergründe der Debatte sei auf den Literaturüberblick verwiesen.

(2) Vgl. für nöhere Informationen die VöW-Mitteilungen in diesem Heft.

\section{Der Autor}

Dr. Uwe Schneidewind ist Vorsizzender der Vereinigung für ökologische Wirtschafisforschung IVÖW e.V.).

Kontakr: VÖW-Geschäftsstelle, Giesebrechistr. 13, 10629 Berlin, Tel. 030/8851800 
(c) 20I0 Authors; licensee IÖW and oekom verlag. This is an article distributed under the terms of the Creative Commons Attribution Non-Commercial No Derivates License (http://creativecommons.org/licenses/by-nc-nd/3.o/), which permits unrestricted use, distribution, and reproduction in any medium, provided the original work is properly cited. 